\title{
Is phage therapy suitable for treating chronic sinusitis Staphylococcus aureus infection?
}

\author{
Peter G Speck*,1 \& Peter-John Wormald ${ }^{2}$ \\ ${ }^{1}$ College of Science \& Engineering, Flinders University, Bedford Park, South Australia \\ ${ }^{2}$ Department of Surgery-Otolaryngology Head \& Neck Surgery, University of Adelaide, Adelaide, South Australia \\ *Author for correspondence: peter.speck@flinders.edu.au
}

'The extensive use of antibiotics, proper and improper, has provided selective pressure on bacteria to evolve toward resistance. The therapeutic use of bacteriophages ('phages' - viruses that infect and can kill bacteria) is an old idea arousing new interest, which may represent a viable alternative to antibiotics in some settings."

First draft submitted: 7 November 2017; Accepted for publication: 11 December 2017; Published online: 24 May 2018

Keywords: bacteriophage $\bullet$ chronic sinusitis $\bullet$ Staphylococcus aureus

The extensive use of antibiotics, proper and improper, has provided selective pressure on bacteria to evolve toward resistance. The common opportunistic bacterial pathogen Staphylococcus aureus is notorious for resisting a broad variety of antibiotics, especially in biofilm form. One clinical setting in which this bacterium, and its antibiotic resistance, is problematic is in chronic sinus infections, which are associated with chronic rhinosinusitis (CRS). In the USA, where the population prevalence of CRS is thought to be as high as $16 \%$ [1], its direct annual cost to healthcare systems is estimated at over USD $\$ 10$ billion, and its indirect cost due to work-related productivity losses is in excess of USD $\$ 20$ billion [2]. The presence of $S$. aureus biofilms in CRS is associated with a number of adverse outcomes and increased disease severity, such as more frequent out-patient visits [3], increased risk of recurrent infections and antibiotic use [4] as well as poorer progression following sinus surgery $[5,6]$.

It is vital that new therapies are introduced that effectively treat antibiotic-resistant infections, especially in view of the paucity of new antibiotics in the product development pipeline [7]. The therapeutic use of bacteriophages ('phages' - viruses that infect and can kill bacteria) is an old idea arousing new interest, which may represent a viable alternative to antibiotics in some settings. However, there are issues surrounding the use of phages as an alternative or adjunctive therapy for bacterial CRS that must be considered, and these are discussed below.

\section{Safety}

There is abundant evidence that supports the safety of phages. There is a long history of phage use within former USSR countries, with few, if any, reports of adverse reactions [8]. Phages only infect bacteria, and are the most common biological entity in the biosphere and are present in many environments. In the gut, they vastly outnumber bacteria [9] and seawater contains $>10^{8}$ phage particles/ml [10]. In 2006, the US FDA gave 'generally regarded as safe' status to the phage preparation Listex, against Listeria monocytogenes, to allow its addition to human foods. In a sheep model of CRS, administration of $S$. aureus phages has been shown to be safe and well tolerated [11,12].

A clinical trial has been conducted with the AmpliPhi Biosciences Corporation phage product AB-SA01, a cocktail of three lytic phages against $S$. aureus, on CRS patients (Australia New Zealand Clinical Trial Registry ID ACTRN12616000002482). This trial was undertaken with patients who had undergone sinus surgery, so access to the sinuses was readily available for drug administration via the nares. This trial showed the phage product to be safe, and yielded preliminary data consistent with it bringing about a reduction in patient symptoms and $S$. aureus load (manuscript in preparation).

An advantage of phages is that they deliver selective toxicity, that is, they typically target a single bacterial species, so they are not expected to cause the damage to the human microbiome that results from broad-spectrum antibiotics. 


\section{Efficacy}

There is approaching 100 years of history of phage therapy having been used efficaciously in a wide variety of settings [13]. In a sheep model of CRS, S. aureus phages effectively reduce the load of $S$. aureus, including in biofilm form $[11,14]$. There seems little doubt that phages have great promise as an effective treatment of bacterial infections of the sinuses.

\section{Will resistance to phages emerge?}

Just as bacteria can evolve resistance to antibiotics, they can also erect barriers to phages, so it is important that the therapeutic opportunity offered by phages is not squandered by misuse, as has occurred with antibiotics. Bacteria employ a large number of resistance mechanisms, as reviewed by Labrie et al. [15], and on exposure to a single population of phages, resistance readily emerges. Bacterial resistance mechanisms include prevention of phage adsorption, such as the blockade of phage receptors by protein A as seen in S. aureus; prevention of phage DNA entry into cells, such as the Sie systems in some Gram-negative bacteria; cutting phage nucleic acids, for example, by restriction enzymes, or by CRISPR/Cas systems in which short DNA sequences from phages are incorporated in the bacterial genome providing a primitive immune system that can cut exogenous DNA and abortive infection systems, such as the Rex proteins in E. coli [15].

The use of phage mixtures or 'cocktails' has been shown to reduce the potential development of resistant bacteria, as reviewed by Chan et al. [16], suggesting that single phage preparations should be avoided for phage therapy. Protocols to prevent misuse of antibiotics, such as seen in modern antibiotic stewardship, should be adapted to achieve the same goal in the use of phages.

\section{Potential for phage therapy in combination with antibiotics}

There is promising evidence that the combination of phages and antibiotics may yield synergistic effects in treating bacterial infections. For example, the Pseudomonas aeruginosa lytic phage OMKO1 uses the outer membrane porin $\mathrm{M}$ of the multidrug efflux systems MexAB and MexXY as a receptor, therefore bacterial evolution to resist attack by this phage requires changes to efflux pump mechanisms, bringing increased susceptibility to some antibiotics [17]. Thus, the combined evolutionary pressures of phage attack together with antibiotics may restore susceptibility to antibiotics, a potentially attractive clinical option. This view is supported by the recent successful case of phage therapy in a patient in CA, USA, who was suffering from a severe disseminated multidrug-resistant Acinetobacter baumanii infection. The patient had deteriorated over a 4-month infection period and had a poor prognosis. Treatment was administered by intravenous infusion of a total of nine $A$. baumannii phages. Over an 8-day period, the target bacteria developed resistance to all phages, but had reacquired antibiotic susceptibility. The dual modes of actions of antibiotic/phage therapy lead to the patient's recovery [18].

\section{Sinus anatomy: can phage products be readily introduced into sinuses?}

The sinus ostium (the opening from the nasal cavity to the sinuses) is sufficiently small that it is not reliably penetrated by sinus irrigant - potentially the vehicle for phages - in patients who have not undergone sinus surgery [19]. Thus, the need for prior sinus surgery is a potential barrier for the use of phages in treating CRS.

\section{Phage host range}

Many phages display a narrow host range, that is, they only infect a subset of strains of their target bacterial species. However, phages against $S$. aureus have been isolated that display a broader host range, so that they are lytic against many different strains and clinical isolates of their target species [14]. The use of a cocktail of such S. aureus phages potentially yields a phage product that can target $>90 \%$ of clinical isolates of $S$. aureus worldwide. Phages for use in therapy must be selected with their host range in mind.

\section{Other barriers to introduction of phage therapy}

The effects of phages on local microbiomes remain poorly studied. It is possible that removal of a pathogen such as $S$. aureus could allow for other opportunistic bacterial species present in the microbiome of a CRS patient to become dominant and pathogenic. Clinical trials conducted with phages should consider the examination of microbiomes before and after phage therapy. Current thinking is that CRS cases are typically associated with a single predominant bacterial pathogen, for example, $S$. aureus as described here. If microbiome studies show that other pathogens can opportunistically expand and fill the void left by phage therapy, then there are a number of approaches that 
could be adopted, including use of phage cocktails targeting multiple bacterial species. An alternative would be genetic engineering of phages to achieve this goal. The engineering of 'designer' phages, potentially with superior therapeutic capability, is being attempted by numerous research groups.

Although the isolation and propagation of lytic phages against bacteria associated with CRS might appear to be a relatively simple undertaking, regulatory agencies require phage products manufactured for human use to reach current good manufacturing practice (cGMP) standards, which require considerable investment and expense.

Regardless of the success of phages in the laboratory or animal tests, any promising approach must undergo clinical trials. These are expensive, typically many millions of pounds, and are beyond the resources of many small biotech companies [20]. The investment and effort by pharmaceutical companies in the development of new antimicrobials has decreased dramatically in the last 20 years. A 2009 survey of 13 large pharmaceutical companies found just three new antimicrobial compounds in advanced development [21]. With large companies directing their resources elsewhere, governments and funding agencies must encourage the development of promising new antimicrobial strategies, including phages, alongside supporting clinical trials of these new agents. Until synergistic support from researchers, pharmaceutical companies and the government is achieved, development of new and effective antimicrobial agents for use in CRS will continue to face this critical blockage.

\section{Financial \& competing interests disclosure}

The authors have no relevant affiliations or financial involvement with any organization or entity with a financial interest in or financial conflict with the subject matter or materials discussed in the manuscript. This includes employment, consultancies, honoraria, stock ownership or options, expert testimony, grants or patents received or pending, or royalties.

No writing assistance was utilized in the production of this manuscript.

\section{References}

1. Halawi AM, Smith SS, Chandra RK. Chronic rhinosinusitis: epidemiology and cost. Allergy Asthma Proc. 34(4), 328-334 (2013).

2. Rudmik L. Economics of chronic rhinosinusitis. Curr. Allergy Asthma Rep. 17(4), 20 (2017).

3. Singhal D, Foreman A, Jervis-Bardy J, Wormald PJ. Staphylococcus aureus biofilms: nemesis of endoscopic sinus surgery. Laryngoscope 121(7), 1578-1583 (2011).

4. Jervis-Bardy J, Wormald PJ. Microbiological outcomes following mupirocin nasal washes for symptomatic, Staphylococcus aureus-positive chronic rhinosinusitis following endoscopic sinus surgery. Int. Forum Allergy Rhinol. 2(2), 111-115 (2012).

5. Foreman A, Wormald PJ. Different biofilms different disease? A clinical outcomes study. Laryngoscope 120(8), 1701-1706 (2010).

6. Singhal D, Psaltis AJ, Foreman A, Wormald PJ. The impact of biofilms on outcomes after endoscopic sinus surgery. Am. J. Rhinol. Allergy 24(3), 169-174 (2010).

7. Charles PGP, Grayson ML. The dearth of new antibiotic development: why we should be worried and what we can do about it. Med. J. Aust. 181(10), 549 (2004).

8. Sulakvelidze A, Alavidze Z, Morris JG Jr. Bacteriophage therapy. Antimicrob. Agents Chemother. 45(3), 649-659 (2001).

9. Reyes A, Semenkovich NP, Whiteson K, Rohwer F, Gordon JI. Going viral: next-generation sequencing applied to phage populations in the human gut. Nat. Rev. Microbiol. 10(9), 607-617 (2012).

10. Suttle CA. Marine viruses-major players in the global ecosystem. Nat. Rev. Microbiol. 5(10), 801-812 (2007).

11. Drilling A, Morales S, Boase $S$ et al. Safety and efficacy of topical bacteriophage and ethylenediaminetetraacetic acid treatment of Staphylococcus aureus infection in a sheep model of sinusitis. Int. Forum Allergy Rhinol. 4(3), 176-186 (2014).

12. Drilling AJ, Ooi ML, Miljkovic D et al. Long-term safety of topical bacteriophage application to the frontal sinus region. Front. Cell Infect. Microbiol. 7, 49 (2017).

13. Abedon ST, Kuhl SJ, Blasdel BG, Kutter EM. Phage treatment of human infections. Bacteriophage 1(2), 66-85 (2011).

14. Drilling A, Morales S, Jardeleza C, Vreugde S, Speck P, Wormald PJ. Bacteriophage reduces biofilm of Staphylococcus aureus ex vivo isolates from chronic rhinosinusitis patients. Am. J. Rhinol. Allergy. 28(1), 3-11 (2014).

15. Labrie SJ, Samson JE, Moineau S. Bacteriophage resistance mechanisms. Nat. Rev. Microbiol. 8(5), 317-327 (2010).

16. Chan BK, Abedon ST, Loc-Carrillo C. Phage cocktails and the future of phage therapy. Future Microbiol. 8(6), 769-783 (2013).

17. Chan BK, Sistrom M, Wertz JE, Kortright KE, Narayan D, Turner PE. Phage selection restores antibiotic sensitivity in MDR Pseudomonas aeruginosa. Sci. Rep. 6, 8 (2016).

18. Schooley RT, Biswas B, Gill JJ et al. Development and use of personalized bacteriophage-based therapeutic cocktails to treat a patient with a disseminated resistant Acinetobacter baumannii infection. Antimicrob. Agents Chemother. 61(10), 14 (2017). 


\section{Editorial Speck \& Wormald}

19. Grobler A, Weitzel EK, Buele A et al. Pre- and postoperative sinus penetration of nasal irrigation. Laryngoscope 118(11), 2078-2081 (2008).

20. Cole ST. Who will develop new antibacterial agents? Philos. Trans. R. Soc. Lond. B. Biol. Sci. 369(1645), 20130430 (2014).

21. Boucher HW, Talbot GH, Bradley JS et al. Bad bugs, no drugs: no ESKAPE! an update from the Infectious Diseases Society of America. Clin. Infect. Dis. 48(1), 1-12 (2009). 